\title{
KiSS-1-mediated suppression of the invasive ability of human pancreatic carcinoma cells is not dependent on the level of KiSS-1 receptor GPR54
}

\author{
CHUN-HUI WANG ${ }^{1 *}$, CHONG QIAO ${ }^{2 *}$, RUO-CHEN WANG ${ }^{3}$ and WEN-PING ZHOU ${ }^{1}$ \\ ${ }^{1}$ Department of Hepatobiliary Surgery, General Hospital of Shenyang Military Region, Shenyang, Liaoning 110016; \\ ${ }^{2}$ Department of Obstetrics and Gynecology, Shengjing Hospital, China Medical University, Shenyang, Liaoning 110004; \\ ${ }^{3}$ Liaoning Province Shiyan High School, Shenyang, Liaoning 110841, P.R. China
}

Received November 3, 2014; Accepted July 22, 2015

DOI: $10.3892 / \mathrm{mmr} .2015 .4535$

\begin{abstract}
The onset of local invasion and lymphatic metastasis in pancreatic cancer limits survival following surgical intervention and additional therapies. Reduced expression of KiSS-1 in pancreatic cancer is associated with cancer metastasis. Previous studies have indicated that kisspeptin, the KiSS-1 peptide, is able to bind to its receptor-GPR54 (hOT7T175) and suppress the migration of PANC-1 pancreatic cancer cells. Whether the metastatic suppression of KiSS-1 is dependent on the levels of GPR54 in pancreatic cancer cell lines remains unclear. Human BxPC-3 pancreatic carcinoma cells are highly differentiated without exhibiting metastasis, however PANC-1 pancreatic carcinoma cells are poorly differentiated and exhibit local and lymph node metastasis. Compared with primary cultured trophoblasts, BxPc-3 and PANC-1 cells were observed to express low levels of KiSS-1 mRNA and protein, measured using reverse transcription-quantitative polymerase chain reaction and western blotting, respectively. However, greater mRNA and protein expression levels of GPR54 were observed in PANC-1 cells compared with BxPc-3 cells. An MTT assay was used to investigate the effect of KiSS-1 on BxPc-3 and PANC-1 cell proliferation. There were no significant differences in proliferation following transfection with KiSS-1 in BxPc-3 and PANC- 1 cells compared with the controls $(\mathrm{P}>0.05)$. A Transwell assay with chambers coated with Matrigel was used to evaluate the in vitro invasive ability of $\mathrm{BxPc}-3$ and PANC-1 cells, with the invasion index of $\mathrm{BxPc}-3$ and
\end{abstract}

Correspondence to: Dr Chun-Hui Wang, Department of Hepatobiliary Surgery, General Hospital of Shenyang Military Region, 83 Wenhua Road, Shenyang, Liaoning 110016, P.R. China Email: wangchh_2013@hotmail.com

*Contributed equally

Key words: KiSS-1, pancreatic cancer, GPR54, proliferation, invasion
PANC-1 cells significantly reduced following 48 h of KiSS-1 overexpression $(\mathrm{P}<0.05)$. The mRNA and protein expression levels of KiSS-1 were significantly increased in BxPc-3 and PANC-1 cells $48 \mathrm{~h}$ subsequent to transfection with KiSS-1 $(\mathrm{P}<0.05)$, while GPR54 expression was not altered $(\mathrm{P}>0.05)$. KiSS-1 is a metastasis suppressor gene of pancreatic cancer, and this suppression is not dependent on the expression levels of GPR54. Therefore, KiSS-1 is potentially a novel target for gene therapy.

\section{Introduction}

Pancreatic cancer is a major cause of mortality in China, and its incidence has increased in the last decade, with incidence approaching that of Western countries. At present, pancreatic cancer remains the fourth leading cause of cancer-associated mortality in the USA, and the 5-year relative survival rate is approximately $7.2 \%$ (all stages included) $(1,2)$.

If the tumor is resectable, surgical resection is the only definitive option for treatment of pancreatic cancer (3). Pancreatic cancer is characterized by insidious onset, high local invasiveness and early metastasis (4). Greater than $90 \%$ of patients with pancreatic cancer present with local invasion and overt metastasis at the time of the occurrence of clinical symptoms and diagnosis (5). These are the key factors for the failure of treatment aiming to prevent recurrence and cancer-associated mortality (5). The 1-year survival rate of patients who suffer from metastatic pancreatic cancer is lower than $20 \%$ and the majority of patients do not live for longer than 2 years following diagnosis (6). The survival rate has not improved substantially during the last 40 years, despite the use of surgery, chemotherapy and radiation therapy (7). Therefore, understanding the metastatic mechanisms of pancreatic cancer and targeted gene therapy for metastasis is important. Previous studies have investigated the expression of KiSS-1 and its peptide, metastin/kisspeptin, in pancreatic cancer. It has been observed that KiSS-1 and kisspeptin are expressed in normal pancreatic tissue, and the reduced expression levels of KiSS-1 and kisspeptin are negatively correlated with TNM stage, invasion and metastasis of pancreatic cancer (8-11). Studies have indicated that 
kisspeptin binds to its receptor GPR54 (hOT7T175) and suppresses the migration of PANC-1 pancreatic cancer cells, and activates ERK1 (9,11-14). However, the mechanism of KiSS-1-mediated suppression of metastasis in pancreatic cancer remains unclear. In previous studies, eukaryotic expression plasmids of KiSS-1 were cloned and constructed from human pancreatic tissue $(15,16)$. The present study aimed to investigate whether the metastatic suppression of KiSS-1 on pancreatic cancer cells was dependent on the GPR54 expression levels in pancreatic cancer cell lines.

\section{Materials and methods}

Materials and reagents. The pancreatic cancer cell lines, BxPC-3 and PANC-1, were obtained from the American Type Culture Collection (Manassas, VA, USA). Dulbecco's modified Eagle's medium (DMEM; cat. no. 41965-062), DMEM/F12 (cat. no. 11320-082), fetal bovine serum (FBS; cat. no. 26140-079), TRIzol ${ }^{\circledR}$ Reagent (cat. no. 15596-018), SuperScript ${ }^{\circledR}$ II Reverse Transcriptase (cat. no. 18064-014) and Lipofectamine ${ }^{\circledR} 2000$ Transfection Reagent (cat.no. 11668-019) were obtained from Invitrogen Life Technologies (Beijing, China). The iQ SYBR ${ }^{\circledR}$ Green Super mix (cat. no. 1708882) was purchased from Bio-Rad Laboratories, Inc. (Shanghai ,China), bicinchoninic acid (BCA) protein assay kit (cat. no. 23227) was obtained from Thermo Fisher Scientific (Shanghai, China); polyclonal goat anti-human GPR54 (N-14) (cat. no. sc-48219) and goat anti-rabbit IgG (cat. no. sc-2004) and donkey anti-goat IgG (cat. no. sc-2005) horseradish peroxidase-labeled secondary antibodies were purchased from Santa Cruz Biotechnology, Inc. (Dallas, TX, USA). Polyclonal rabbit anti human metastin/kisspeptin (1-54) (cat. no. G-048-59) was purchased from Phoenix Pharmaceuticals, Inc. (Burlingame, CA, USA). Polyclonal rabbit anti- $\beta$-actin (cat. no. ab8227) was purchased from Abcam (Pak Shek Kok, Hong Kong). Pierce Enhanced Chemiluminescence (ECL) Western Blotting kit (cat. no. 32109) was obtained from GE Healthcare Bio-Sciences (Pittsburgh, PA, USA). The Vectastain ${ }^{\circledR}$ Elite ABC kit (cat. no. PK-6200) was obtained from Vector Laboratories (Burlingame, CA, USA) and BioCoat ${ }^{\mathrm{TM}}$ Matrigel $^{\mathrm{TM}}$ invasion chambers in two 24-well plates $(8 \mu \mathrm{m}$ pore-size) were purchased from BD Biosciences (San Jose, CA, USA). The pcDNA3 vector was obtained from Invitrogen Life Technologies (Carlsbad, CA, USA) and pcDNA3/KISS-1 was produced as previously described (15).

Cell culture and transfection. The PxPC-3 and PANC-1 pancreatic cancer cell lines were cultured in DMEM supplemented with $10 \%$ FBS, $1 \%$ penicillin and $1 \%$ streptomycin. Human trophoblast cells were obtained from legal abortions (6-12 weeks of gestational age), with the approval of the local ethical committee (in compliance with the Helsinki Declaration) and the consent of the participating patients. Trophoblast cells were isolated as described previously $(17,18)$. The primary trophoblast cells were cultured in DMEM/F12 medium supplemented with $20 \%$ FBS, $1 \%$ penicillin and $1 \%$ streptomycin. Cells were cultured at $37^{\circ} \mathrm{C}$ in a humidified incubator with $5 \% \mathrm{CO}_{2}$. The primary trophoblast cells were used as the control for the relative mRNA expression of KiSS-1 and GPR54.
At $12 \mathrm{~h}$ prior to transfection, cells were seeded into 6-well plates at a density of $5 \times 10^{5}$ cells/well. Cells were transfected when plate confluence had reached $80-90 \%$. The cells were transfected with $3.3 \mu \mathrm{g} /$ well PcDNA3.1-KISS-1 vector using $45 \mu \mathrm{l}$ Lipofectamine ${ }^{\circledR} 2000$. Following $6 \mathrm{~h}$ of incubation at $37^{\circ} \mathrm{C}$, the plasmid containing medium was replaced with normal cell culture medium. Cells were transfected with the empty pcDNA3 vector as a negative control. All transfections were performed in triplicate for each time point.

RNA extraction and reverse transcription-quantitative polymerase chain reaction ( $R T-q P C R)$. RT-qPCR was conducted as previously described with minor modifications (18). Briefly, total RNA was extracted from untransfected and transfected BxPC-3, PANC-1 and trophoblast cells using TRIzol ${ }^{\circledR}$ Reagent. RNA (500 ng) was converted to cDNA using SuperScript ${ }^{\circledR}$ II Reverse Transcriptase. RT-qPCR analysis was performed using the ABI PRISM ${ }^{\circledR} 7700$ Sequence Detector (Applied Biosystems Life Technologies, Foster City, CA, USA). RT-qPCR was conducted using the reagents and instructions of the iQ SYBR Green Super mix. The PCR primers for human genes KiSS-1 (NM_002256), GPR54 (NM_032551) and the internal control $\beta$-actin (NM_001101) are as previously described (18): KiSS-1, forward ACTCAC TGGTTTCTTGGCAGCT and reverse CAGAGGCCACCT TTTCTAATGG; GPR54, forward CGACTTCATGTGCAA GTTCGTC and reverse CACACTCATGGCGGTCAGAG; $\beta$-actin, forward ACCAACTGGGACGACATGGAGAAAA and reverse TACGGCCAGAGGCGTACAGGGATAG. The PCR was performed for $60 \mathrm{sec}$ at $95^{\circ} \mathrm{C}$ followed by 40 cycles of $15 \mathrm{sec}$ denaturation at $95^{\circ} \mathrm{C}$ and $60 \mathrm{sec}$ annealing at $60^{\circ} \mathrm{C}$. The RT-qPCR reaction was conducted in triplicate in a final volume of $25 \mu \mathrm{l}$ with $100 \mathrm{ng}$ cDNA. The quantity of cDNA for each experimental gene was normalised to the quantity of $\beta$-actin cDNA in each sample. Relative expression was determined using the $\Delta \Delta \mathrm{Ct}$ (threshold cycle) method according to the manufacturer's instructions.

Western blot analysis. Western blotting was conducted as described previously (18). Briefly, protein extracts were prepared from cells by adding modified RIPA lysis buffer supplemented with protease inhibitor cocktail tablets (Roche Diagnostics, Basel, Switzerland). Protein concentrations were quantified using the BCA protein assay. Protein samples $(30 \mu \mathrm{g})$ were migrated on a $15 \%$ sodium dodecyl sulfate polyacrylamide gel electrophoresis and transferred onto a polyvinylidene difluoride membrane (GE Healthcare Bio-Sciences). The membrane was blocked with blocking buffer (Tris-buffered saline with Tween 20 with 5\% non-fat milk) and incubated with the primary antibody. The following primary antibodies were used: Rabbit anti-human metastin/kisspeptin (1-54) (1:800), goat anti-human GPR54 (N-14) (1:500) and polyclonal rabbit anti- $\beta$-actin $(1: 1,000)$. Endogenous $\beta$-actin expression served as an internal control. Primary antibody binding was detected using the following secondary antibodies: Anti-rabbit IgG and anti-goat IgG antibody conjugated to horseradish perioxidase $(1: 10,000$; Santa Cruz Biotechnology). Detection was achieved using the ECL Western Blotting kit (GE Healthcare Bio-Sciences) and X-ray film (Kodak, Shanghai, China). 
Cell proliferation assay. This method provides a quantitative measurement of the number of cells with metabolically active mitochondria and is based on the mitochondrial reduction of a tetrazolium bromide salt [MTT assay; 3-(4,5-dimethylthiazol-2)-2, 5-diphenyltetrazolium bromide; cat. no. M5655, Sigma-Aldrich, St. Louis, MO, USA]. Cells were seeded at a density of $3 \times 10^{3}$ cells/well in a 96-well plate and cultured in the presence of $10 \%$ FBS for $0,1,2,3,4,5$ and 6 days. The cells were pulsed with MTT $20 \mu \mathrm{l} / \mathrm{well}(5 \mathrm{mg} / \mathrm{ml}$ in phosphate-buffered saline for $4 \mathrm{~h}$. The purple-blue MTT formazan precipitate was dissolved in $100 \mu 1$ of dimethyl sulfoxide and agitated for $10 \mathrm{~min}$. Absorbance was measured at $490 \mathrm{~nm}$ with a Beckman-DU 640 Spectrophotometer (Beckman Coulter, Inc., Brea, CA, USA). Experiments were repeated six times.

Invasion assays. For the invasion assays, BioCoat ${ }^{\mathrm{TM}}$ Matrigel ${ }^{\circledR}$ invasion chambers in two 24-well plates with polycarbonate filters $(8 \mu \mathrm{m})$ were used. Cells $\left(5 \times 10^{4}\right.$ cells $\left./ \mathrm{ml}\right)$ in $500 \mu \mathrm{l}$ complete medium were seeded into the upper chamber. Next, $600 \mu \mathrm{l}$ complete medium was added to the lower chamber, and the plate was incubated at $37^{\circ} \mathrm{C}$ in a $5 \% \mathrm{CO}_{2}$ incubator for $48 \mathrm{~h}$. Cells on the lower surface of the filter were stained with hexamethylpararosaniline and counted under a light microscope (Nikon Eclipse Ci-S; Nikon Instruments Inc., Shanghai, China). Each group of cells, which included BxPC-3 (blank control), BxPC-3/vector (negative control), BxPC-3/pcDNA3/KiSS-1, PANC-1 (blank control), PANC-1/vector (negative control) and PANC-1/pcDNA3/KiSS-1, was seeded in three wells and the experiment was performed in triplicate for these two different cell lines. Invaded cells were counted in five randomly selected fields for each filter under a light microscope with 100x magnification (Nikon Eclipse Ci-S). The invasion index was defined as follows: (Number of cells that migrated through the $8 \mu \mathrm{m}$ pores of the filter in the experimental group/number of cells that migrated through the filter in the blank control group) $\mathrm{x} 100$.

Statistical analysis. All experiments were conducted a minimum of three times. Within each experiment, a minimum of three replicates were used. Data are presented as the mean \pm standard deviation. Data were analyzed using SPSS software, version 16.0 (SPSS, Inc., Chicago IL, USA). Analysis of variance was conducted followed by Student's t-test. $\mathrm{P}<0.05$ was considered to indicate a statistically significant difference.

\section{Results}

Expression of KiSS-1 and its receptor GPR54 in human pancreatic cancer cell lines. The human pancreatic ductal adenocarcinoma cell lines BxPc-3 (CRL-1687) and PANC-1 (CRL-1469) were obtained from the American Type Culture Collection and were grown as recommended. BxPc-3 cells were originally derived from a 61-year-old Caucasian female with a well differentiated pancreatic carcinoma without any evidence of metastasis. PANC-1 cells were derived from a primary pancreatic ductal adenocarcinoma in a 56-year-old Caucasian male that extended to involve the duodenal wall and had metastasized to one peripancreatic lymph node.

To determine the expression levels of KiSS-1 and its receptor GPR54 in pancreatic ductal adenocarcinoma, the levels of KiSS-1 and GPR54 mRNA were measured using RT-qPCR in cultured pancreatic ductal adenocarcinoma cells. Compared with the primary cultured human trophoblast cells, KiSS-1 mRNA was expressed at reduced levels in BxPc-3 and PANC-1 cells, and the relative expression of KiSS-1 mRNA was $10 \%$ of that in human trophoblast cells (Fig. 1A). The protein levels of kisspeptin and GPR54 were measured using western blotting. The expression level of kisspeptin was observed to be low in BxPc-3 and PANC-1 cells compared with trophoblast cells (Fig. 1B and C). GPR54 mRNA expression was reduced in BxPc-3 cells, with levels $15.9 \%$ of that in human trophoblasts. However, the expression of GPR54 mRNA in PANC-1 cells was $92.7 \%$ of that in human trophoblast cells, 5.83 fold higher than in $\mathrm{BxPc}-3$ cells (Fig. 1A). The protein expression level of GPR54 was significantly higher in PANC-1 cells than that in BxPc-3 cells, as detected by western blotting (Fig. 1D).

Transfection of KiSS-1 increases expression of KiSS-1 with no significant alterations in GPR54 expression. Following transfection of BxPc-3 and PANC-1 cells with pcDNA3-KiSS-1, an increase in the expression levels of KiSS-1 mRNA (Fig. 2A and C) and kisspeptin (Fig 2B and D) was observed (P<0.05; Fig. 2).

The expression of GPR54 in BxPc-3 and PANC-1 cells transfected with pcDNA3-KiSS-1 was not altered compared with control cells ( $\mathrm{P}>0.05$; Fig. 2).

Overexpression of KiSS-1 does not alter cellular proliferation in BxPc-3 and PANC-1 cells. The effect of KiSS-1 overexpression on the proliferation of pancreatic cancer cells was investigated. Following transfection, the absorbance values $(490 \mathrm{~nm})$ of pcDNA3-KiSS-1-transfected BxPc-3 and PANC-1 cells did not differ from those of control cells $(\mathrm{P}>0.05)$. These results indicate that KiSS-1 overexpression had no effect on the proliferation of BxPc-3 and PANC-1 cells (Fig. 3).

Overexpression of KiSS-1 suppresses cell invasion. The invasive ability of BxPc-3 and PANC-1 cells was measured by counting the number of cells that digested Matrigel and migrated through the $8 \mu \mathrm{m}$ pores in the filter. The invasion of BxPc-3 and PANC-1 cells was observed to be significantly reduced by overexpression of KiSS-1, with invasion indices of $50.8 \pm 4.1 \%$ and $48.3 \pm 4.3 \%$, respectively ( $\mathrm{P}<0.05$; Fig. 4). These data indicate that overexpression of KiSS-1 is capable of inhibiting the invasion of BxPc-3 and PANC-1 cells (Fig. 4).

\section{Discussion}

The onset of local invasion and lymphatic metastasis of pancreatic cancer limits the survival rate following surgical intervention and other therapies $(19,20)$. The majority of cases of pancreatic cancer-associated mortality are due to complications resulting from tumor metastasis rather than as a 
A

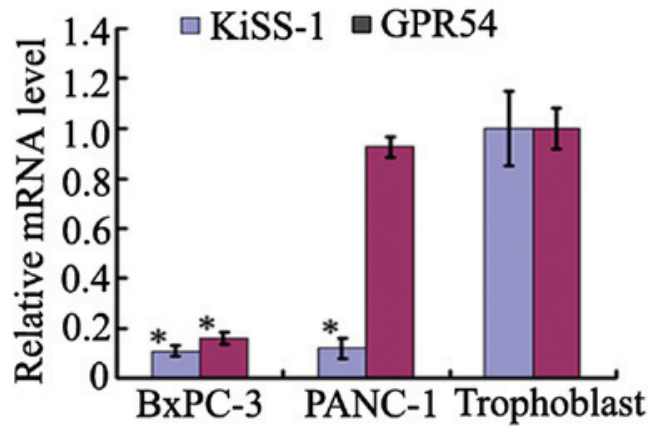

C

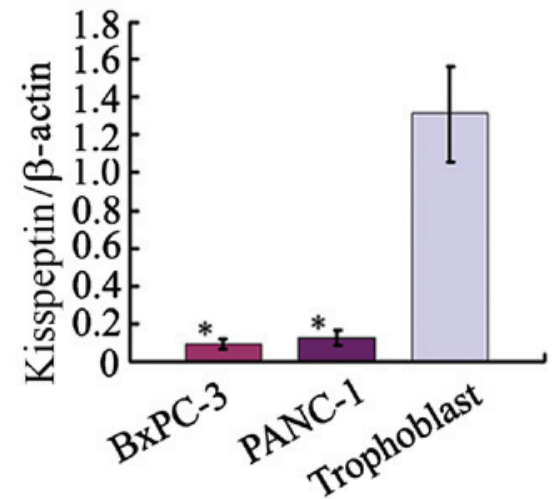

B

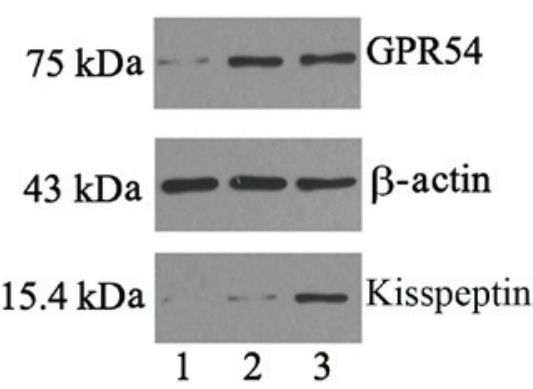

D

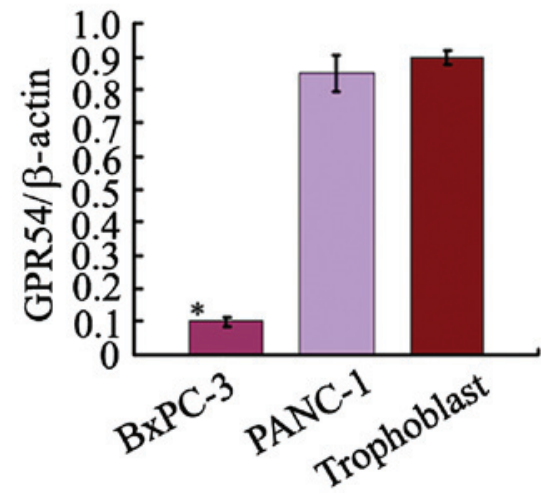

Figure 1. Expression of KiSS-1 and GPR54 in human pancreatic ductal adenocarcinoma cell lines. (A) Reverse transcription-quantitative polymerase chain reaction analysis and (B-D) western blot analysis of KiSS-1 and GPR54 expression in human pancreatic ductal adenocarcinoma cell lines (BxPC-3 and PANC-1) compared with the control (primary cultured human trophoblast cells). (A) BxPC-3 and PANC-1 cells expressed low to undetectable levels of KiSS-1 mRNA. The metastatic pancreatic cancer cell line (PANC-1) expressed greater levels of GPR54, whereas the non-metastatic BxPC-3 cells expressed low levels of GPR54. Levels of the indicated mRNAs (normalized against $\beta$-actin mRNA) from the primary cultured human trophoblast cells were arbitratily set as 1. (B) Representative western blot analysis of kisspeptin and GPR54 protein expression in BxPC-3, PANC-1 and primary cultured human trophoblast cells. Lane 1, BxPC-3 cells; lane 2, PANC-1 cells; lane 3, trophoblast cells. Total proteins (30 $\mu$ g) were separated on a $10 \%$ SDS-PAGE gel and $\beta$-actin was used as a loading control. (C and D) Densitometric analysis of kisspeptin and GPR54 protein expression in BxPC-3, PANC-1 and the primary cultured human trophoblast cells subsequent to normalization to $\beta$-actin. Data represent the mean \pm standard deviation $(n=9)$ following normalization to $\beta$-actin. ${ }^{*} \mathrm{P}<0.05$ vs. primary cultured trophoblasts.

consequence of the original tumor growth. There is increasing interest in understanding the metastatic mechanisms of pancreatic cancer in order to identify possible ways to inhibit local invasion and metastatic cancer progression.

Previous studies have demonstrated that the expression levels of KiSS-1 mRNA in pancreatic cancer are lower compared with normal pancreatic tissue $(8,10)$. Additionally, there are correlations between KiSS-1 mRNA expression levels and clinical stage, metastasis and nerve invasion, without any correlation with histological classification (10). The pancreatic cancer cell line BxPC-3 is highly differentiated without any metastasis whilst PANC-1 is poorly differentiated with local and lymph node metastasis. Based on their different differentiation degrees and metastatic potential, BxPC-3 and PANC-1 cells were selected for the current study.

The mRNA and protein expression levels of KiSS-1 and its receptor GPR54 were measured in $\mathrm{BxPc}-3$ and PANC-1 cells. Numerous previous studies have indicated that KiSS1 and its receptor hOT7T175/GPR54 are expressed in human placental and pancreatic tissues (21-23). Previously, studies have investigated the mRNA and protein expression of KiSS-1 in trophoblasts and the placentas of normal and pre-eclamptic pregnancies, and observed that KiSS-1 is highly expressed $(17,18)$. Therefore, primary cultured trophoblasts were selected as the control for the present study. In the present study, the expression level of KiSS-1 mRNA was observed to be low in BxPc-3 and PANC-1 cells. However, the expression of GPR54 mRNA was higher in PANC-1, with levels comparable to those of human primary cultured trophoblasts, while the expression levels of GPR54 mRNA were low in BxPC-3 cells. Consistent with these results, Masui et al (12) observed low levels of KiSS-1 mRNA expression in BxPC-3, Capan-2, CFPAC-1 and PANC-1 cells, whilst PANC-1 cells demonstrated high expression levels of hOT7T175/GPR54 mRNA

In the present study, the protein levels of KiSS-1 and GPR54 in the BxPC-3 and PANC-1 pancreatic cancer cell lines were investigated using western blotting. This demonstrated that kisspeptin expression was minimal in $\mathrm{BxPC}-3$ and PANC-1 cells. However, the protein level of GPR54 was increased in PANC-1 cells and reduced in BxPC-3 cells. Nagai et al (24) analyzed 53 cases of pancreatic ductal carcinoma and observed that kisspeptin and GPR54 expression was significantly correlated with tumor size, recurrence and survival of patients, however was not correlated with the degree of tissue differentiation. This indicated that kisspeptin may serve a role in the metastasis of pancreatic cancer. In the current study, BxPC-3 cells were selected as a representative cell line with low expression of KiSS-1 and GPR54, whilst PANC-1 cells were a representative cell line with low expression of KiSS-1 and high expression of GPR54. In the current 

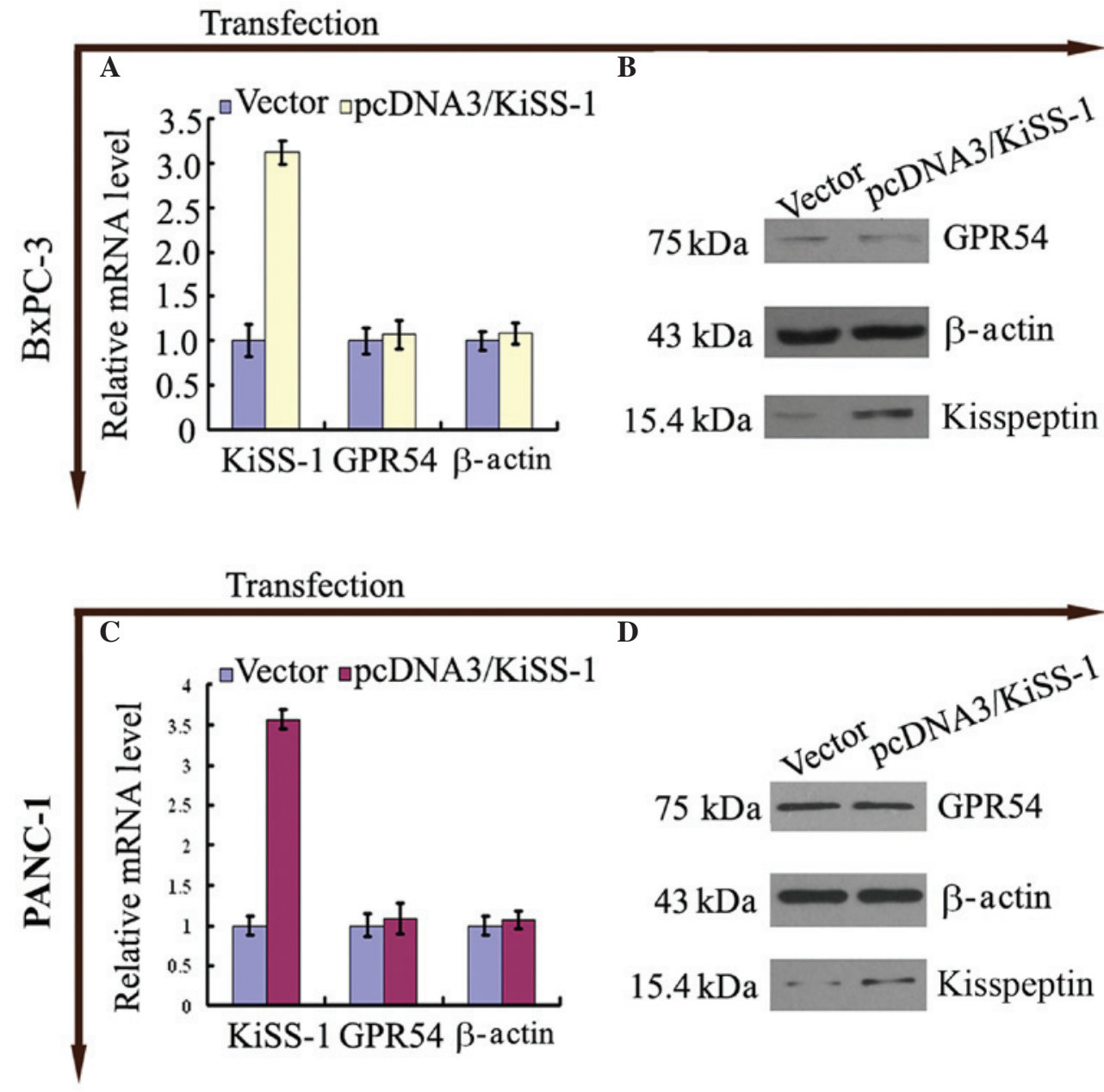

Figure 2. Transfection of the KiSS-1 gene increased expression of KiSS-1 however did not alter GPR54 expression. pcDNA3/KiSS-1 or empty vectors were transfected into (A and B) BxPC-3 or (C and D) PANC-1 cells. Proteins and RNA were extracted $48 \mathrm{~h}$ post-transfection and levels were determined by (A and C) reverse transcription-quantitative polymerase chain reaction and (B and D) western blotting. (A and C) Expression levels of kisspeptin increased 3.2- and 3.5-fold following KiSS-1 overexpression. Expression levels of (A and C) GPR54 mRNA and (B and D) protein were not altered. P<0.05 vs. vector transfection.

study, overexpression of KiSS-1 had no effect on cellular proliferation in BxPC-3 and PANC-1 cells, which is consistent with previous studies in melanoma and breast cancer $(25,26)$. As Masui et al (12) reported, the addition of kisspeptin had no effect on the proliferation of BxPC-3 and PANC-1 cells. Together, this suggests that KiSS-1 and kisspeptin do not affect the proliferative capacity of human pancreatic cancer cell lines.

In the current study, it was demonstrated that the invasive ability of pancreatic cancer cells was significantly reduced following the overexpression of KiSS-1, whilst the empty vector had no effect. It was observed that overexpression of KiSS-1 did not have differential effects upon the two pancreatic cancer cell lines, suggesting that the inhibitory effect on invasion of pancreatic cancer is not dependent on the degree of differentiation. Shirasaki et al (26) observed that the expression of KiSS-1 was lost during the progression of melanocytic tumors in vivo. Jiang et al (27) reported that restoring KiSS-1 expression was able to significantly suppress the metastasis of ovarian cancer and melanoma. The MDA-MB-435 ductal breast carcinoma cell line is metastatic and does not express KiSS-1, however when full-length

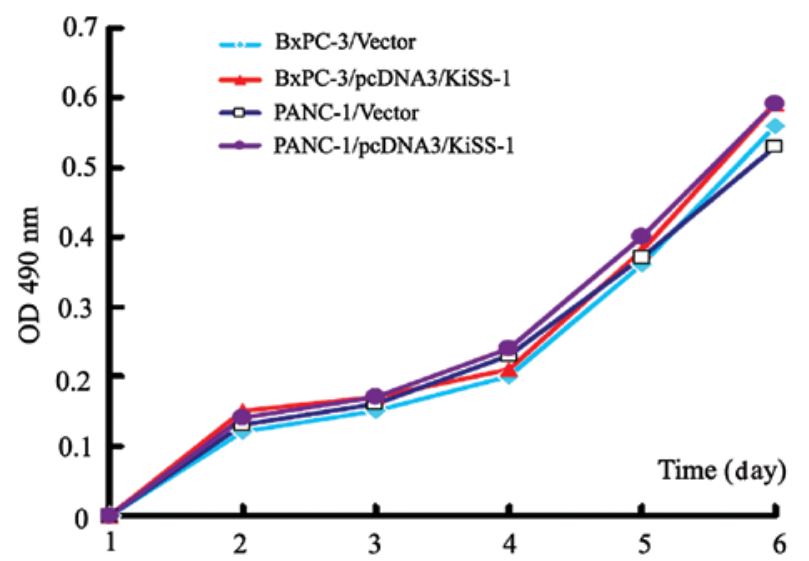

Figure 3. Overexpression of KiSS-1 did not alter cell proliferation in human pancreatic ductal adenocarcinoma cell lines. The proliferation rates were analyzed by growth curves for cells: BxPC-3/vector, BxPC-3/pcDNA3/KiSS-1, PANC-1/vector and PANC-1/pcDNA3/KiSS-1. The cell proliferation rates of BxPC-3 and PANC-1 cells transfected with pcDNA3/KiSS-1 were not significantly altered. OD, optical density.

KiSS-1 cDNA was transfected into MDA-MB-435 cells and injected into the mammary fat pads of athymic nude 


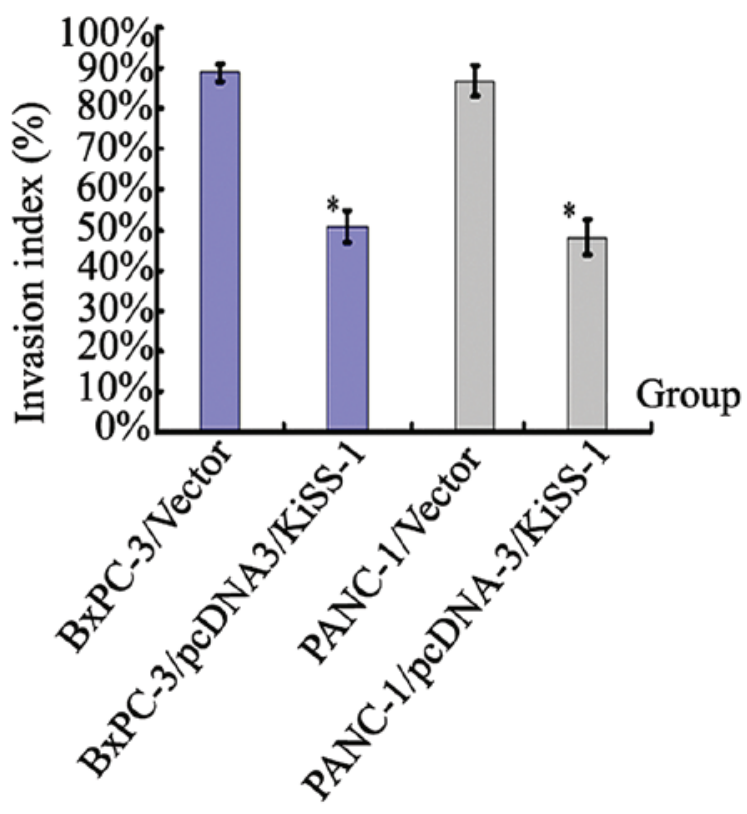

Figure 4. Inhibitive effect of KiSS-1 on the invasion of BxPC-3 and PANC-1 cells in vitro. Invasion index of BxPC-3 and PANC-1 cells transfected by the vector and pcDNA3/KiSS-1. ${ }^{*} \mathrm{P}<0.05$ vs. vector transfection.

mice, lung metastasis was significantly suppressed and the incidence of regional lymph node metastasis was reduced (25). Masui et al (12) examined the effect of exogenous kisspeptin on pancreatic cancer cell proliferation in AsPC-1 and PANC-1 cells, and observed that the migration of AsPC-1 cells was not altered by kisspeptin, while PANC-1 cells were significantly inhibited by kisspeptin. Furthermore, the effect of exogenous kisspeptin on the invasion of these cell lines was associated with the expression level of GPR54/hOT7T175 (12). In a model of melanoma, secretion of KiSS-1 was required for metastasis suppressor activity (28). When KiSS-1 was transfected into a metastatic subclone of the SUIT-2 pancreatic adenocarcinoma cell line, S2VP10, and injected into the tail of the pancreas of severe combined immunodeficiency mice, mice with KiSS-1 expression developed fewer liver and lung metastases than the controls (29). Furthermore, it was observed that the re-expression of KiSS-1 in S2VP10 without expression of GPR54 resulted in suppression of invasion. These observations indicate the existence of an intracrine signaling loop for KiSS-1. Consistent with the results reported by McNally et al (29), the current study also demonstrated that the expression of KiSS-1 mRNA and kisspeptin in BxPC-3 and PANC-1 cells increased following transfection with KiSS-1, whilst the expression level of GPR54 mRNA and protein was unaltered. In the current study, GPR54 was observed exhibit greater expression in PANC-1 cells and lower expression in BxPC-3 cells. Following transfection with KiSS-1, the invasive ability of these cell lines was suppressed, however the proliferative ability was not altered. This suggests that the suppression of invasion mediated by KiSS-1 overexpression was not dependent on the receptor expression levels.

In the intracrine signaling loop, certain cytokines exert their biological function by binding to their receptor in the cytoplasm without the requirement to be secreted out of the cell (30). In this way, certain chemokines and peptide hormones serve an important role in cell apoptosis, migration, invasion and metastasis $(30,31)$. Previous studies have indicated that intracrine signaling occurs in pancreatic adenocarcinoma $(29,32,33)$. Alternatively, there is a possibility that other peptide fragments of KiSS-1, which do not bind GPR54, are released to the extracellular space, however further studies investigating this are required. In an electrophoretic mobility shift assay, KiSS-1 was demonstrated to inhibit the binding of nuclear factor- $\kappa \mathrm{B}$ and the matrix metalloproteinase-9 (MMP-9) promoter, thereby reducing MMP-9 gene transcription and the level of MMP-9, inhibiting the mobility, chemotaxis and invasion of tumor cells (34). It was additionally identified that KiSS-1 gene transfection significantly inhibited MMP-9 gene transcription, and reduced the expression of MMP-9 protein. It is suggested that KiSS-1 suppression of the pancreatic cancer metastasis mechanism is predominantly associated with the inhibition of MMP-9 transcription and the intracellular autocrine loop (34).

In conclusion, the current study demonstrated that increases in KiSS-1 expression suppressed the invasion of pancreatic cancer cells without impacting cellular proliferation. Therefore, targeted gene therapy to increase the expression levels of KiSS-1 and its peptides may be a potential therapeutic strategy for the treatment of pancreatic cancer metastasis.

\section{Acknowledgements}

The current study was supported by grants from the People's Liberation Army Medical Science and Technique Foundation during the 11th Five-Year Plan for Science Technology (Young Scholar Program; grant no. 06Q014), the National Natural Science Foundation of China (General Program; grant no. 81370735) and the Liaoning Provincial Natural Science Foundation (Doctor Startup Fund Program; grant no. 20071038). 


\section{References}

1. Siegel R, DeSantis C, Virgo K, Stein K, Mariotto A, Smith T, Cooper D, Gansler T, Lerro C, Fedewa S, et al: Cancer treatment and survivorship statistics, 2012. CA Cancer J Clin 62: 220-241, 2012.

2. National Cancer Institute: Cancer statistics: SEER stat fact sheets: Pancreas cancer. http://seer.cancer.gov/statfacts/html/pancreas. html. Accessed July 1, 2014.

3. Shaib Y, Davila J, Naumann C, et al: The impact of curative intent surgery on the survival of pancreatic cancer patients: a U.S. population-based study. Am J Gastroenterol 102: 1377-1382, 2007.

4. Kwon D, McFarland K, Velanovich V and Martin RC II: Borderline and locally advanced pancreatic adenocarcinoma margin accentuation with intraoperative irreversible electroporation. Surgery 156: 910-920, 2014.

5. Tuveson DA and Neoptolemos JP: Understanding metastasis in pancreatic cancer: A call for new clinical approaches. Cell 148 21-23, 2012.

6. Jemal A, Bray F, Center MM, Ferlay J, Ward E and Forman D: Global cancer statistics. CA Cancer J Clin 61: 69-90, 2011.

7. Zakharova OP, Karmazanovsky GG and Egorov VI: Pancreatic adenocarcinoma: Outstanding problems. World J Gastrointest Surg 4: 104-113, 2012.

8. Wang C, Qiao C, Ma S, Zhou W and Dai X: Expression of KiSS-1 in human pancreatic cancer and relationship with their invasion and metastasis. China J Mod Med 15: 1620-1623, 1631, 2005.

9. Makri A, Pissimissis N, Lembessis P, Polychronakos C and Koutsilieris M: The kisspeptin (KiSS-1)/GPR54 system in cancer biology. Cancer Treat Rev 34: 682-692, 2008.

10. Wang C, Qiao C and Dai X: Expression of KiSS-1 in human pancreatic cancer and its clinical significance. Chin J Cancer Prev Treat 13: 207-215, 2006

11. Ji K, Ye L, Mason MD and Jiang WG: The Kiss-1/Kiss-1R complex as a negative regulator of cell motility and cancer metastasis (Review). Int J Mol Med 32: 747-754, 2013.

12. Masui T, Doi R, Mori T, Toyoda E, Koizumi M, Kami K, Ito D, Peiper SC, Broach JR, Oishi S, et al: Metastin and its variant forms suppress migration of pancreatic cancer cells. Biochem Biophys Res Commun 315: 85-92, 2004.

13. Szereszewski JM, Pampillo M, Ahow MR, Offermanns S Bhattacharya $\mathrm{M}$ and Babwah AV: GPR54 regulates ERK1/2 activity and hypothalamic gene expression in a $\mathrm{G} \alpha(\mathrm{q} / 11)$ and $\beta$-arrestin-dependent manner. PLoS One 5: e12964, 2010.

14. Francis VA, Abera AB, Matjila M, Millar RP and Katz AA Kisspeptin regulation of genes involved in cell invasion and angiogenesis in first trimester human trophoblast cells. PLoS One 9: e99680, 2014.

15. Wang C, Qiao C and Dai X: Cloning of human KiSS-1 gene and construction of its eukaryotic expression vector. J Clin Med Univ 34: 218-219, 2005 (In Chinese).

16. Li N, Wang HX, Zhang J, Ye YP and He GY: KISS-1 inhibits the proliferation and invasion of gastric carcinoma cells World J Gastroenterol 18: 1827-1833, 2012.

17. Qiao C, Wang $\mathrm{CH}$, Shang T and Lin QD: Clinical significance of KiSS-1 and matrix metalloproteinase-9 expression in trophoblasts of women with preeclampsia and their relation to perinatal outcome of neonates. Zhonghua Fu Chan Ke Za Zhi 40: 585-590, 2005 (In Chinese).

18. Qiao C, Wang C, Zhao J, Liu C and Shang T: Elevated expression of KiSS-1 in placenta of Chinese women with early-onset preeclampsia. PLoS One 7: e48937, 2012.
19. Boyle J, Czito B, Willett C and Palta M: Adjuvant radiation therapy for pancreatic cancer: A review of the old and the new. J Gastrointest Oncol 6: 436-444, 2015.

20. Sinn M, Striefler JK, Sinn BV, Sallmon D, Bischoff S, Stieler JM, Pelzer U, Bahra M, Neuhaus P, Dörken B, et al: Does long-term survival in patients with pancreatic cancer really exist? Results from the CONKO-001 study. J Surg Oncol 108: 398-402, 2013.

21. Muir AI, Chamberlain L, Elshourbagy NA, Michalovich D, Moore DJ, Calamari A, Szekeres PG, Sarau HM, Chambers JK, Murdock P, et al: AXOR12, a novel human G protein-coupled receptor, activated by the peptide KiSS-1. J Biol Chem 276: 28969-28975, 2001.

22. Ohtaki T, Shintani Y, Honda S, Matsumoto H, Hori A, Kanehashi K, Terao Y, Kumano S, Takatsu Y, Masuda Y, et al: Metastasis suppressor gene KiSS-1 encodes peptide ligand of a G-protein-coupled receptor. Nature 411: 613-617, 2001.

23. Lee JH, Miele ME, Hicks DJ, Phillips KK, Trent JM, Weissman BE and Welch DR: KiSS-1, a novel human malignant melanoma metastasis-suppressor gene. J Natl Cancer Inst 88: 1731-1737, 1996.

24. Nagai K, Doi R, Katagiri F, Ito T, Kida A, Koizumi M, Masui T, Kawaguchi Y, Tomita K, Oishi S, et al: Prognostic value of metastin expression in human pancreatic cancer. J Exp Clin Cancer Res 28: 9, 2009.

25. Lee JH and Welch DR: Suppression of metastasis in human breast carcinoma MDA-MB-435 cells after transfection with the metastasis suppressor gene, KiSS-1. Cancer Res 57: 2384-2387, 1997.

26. Shirasaki F, Takata M, Hatta N and Takehara K: Loss of expression of the metastasis suppressor gene KiSS1 during melanoma progression and its association with $\mathrm{LOH}$ of chromosome 6q16.3-q23. Cancer Res 61: 7422-7425, 2001.

27. Jiang Y, Berk M, Singh LS, Tan H, Yin L, Powell CT and Xu Y: KiSS1 suppresses metastasis in human ovarian cancer via inhibition of protein kinase $\mathrm{C}$ alpha. Clin Exp Metastasis 22: 369-376, 2005.

28. Nash KT,PhadkePA, Navenot JM,HurstDR, Accavitti-Loper MA, Sztul E, Vaidya KS, Frost AR, Kappes JC, Peiper SC, et al: Requirement of KISS1 secretion for multiple organ metastasis suppression and maintenance of tumor dormancy. J Natl Cancer Inst 99: 309-321, 2007.

29. McNally LR, Welch DR, Beck BH, Stafford LJ, Long JW, Sellers JC, Huang ZQ, Grizzle WE, Stockard CR, Nash KT, et al: KISS1 over-expression suppresses metastasis of pancreatic adenocarcinoma in a xenograft mouse model. Clin Exp Metastasis 27: 591-600, 2010.

30. Re RN: The origins of intracrine hormone action. Am J Med Sci 323: 43-48, 2002.

31. Gortz A, Nibbs RJ, McLean P, Jarmin D, Lambie W, Baird JW and Graham GJ: The chemokine ESkine/CCL27 displays novel modes of intracrine and paracrine function. J Immunol 169: 1387-1394, 2002

32. Leung PS: The physiology of a local renin-angiotensin system in the pancreas. J Physiol 580: 31-37, 2007.

33. Lau ST and Leung PS: Role of the RAS in pancreatic cancer. Curr Cancer Drug Targets 11: 412-420, 2011.

34. Yan C, Wang H and Boyd DD: KiSS-1 represses 92-kDa type IV collagenase expression by down-regulating NF-kappaB binding to the promoter as a consequence of Ikappa Balpha -induced block of p65/p50 nuclear translocation. J Biol Chem 276: 1164-1172, 2001. 